\title{
Interleukin-11 Attenuates Ifosfamide-Induced Hemorrhagic Cystitis
}

\author{
Jose M. Mota, Gerly A. Brito, Raphael T. Loiola, Fernando Q. Cunha, Ronaldo de A. Ribeiro
}

Departments of Physiology and Pharmacology (JMM, RTL, RAR), and Morphology (GAB), School of Medicine, Federal University of Ceara and Department of Pharmacology FQC), School of Medicine, University of Sao Paulo Ribeirao Preto, Sao Paulo, Brazil

\begin{abstract}
Objective: To investigate the possible protective effect of recombinant human interleukin-11 (rhIL-11) against ifosfamide (IFS)-induced hemorrhagic cystitis (HC).

Materials and Methods: Male Swiss mice (20-30g) were pretreated with rhIL-11 (25-625 $\mu \mathrm{g}$, subcutaneously.) 30 min before intraperitoneal injection of IFS (400 mg/kg) or with saline (control group). Twelve hours later, HC was evaluated by bladder wet weight (BWW) to quantify edema, Evans blue extravasation (EBE) to measure vascular permeability, and macroscopic and microscopic analysis. All bladders were assessed by histopathological analysis.

Results: rhIL-11 (at 125 and $625 \mu \mathrm{g}$ ) attenuated the IFS- induced increase of BWW $(37.48 \%$ and $45.44 \%$, respectively, p < $0.05)$ and $\operatorname{EBE}(62.35 \%$ and $56.47 \%$, respectively, $\mathrm{p}<0.05)$. IFS- induced macroscopic edema and hemorrhage and microscopic alterations, were also prevented by rhIL-11 at $625 \mu \mathrm{g}$. $(\mathrm{p}<0.05)$.

Conclusion: Our results demonstrate a protective effect of rhIL-11 on experimental IFS- induced HC, not previously reported.
\end{abstract}

Key words: bladder; ifosfamide; cystitis; interleukin-11; rats

Int Braz J Urol. 2007; 33: 704-10

\section{INTRODUCTION}

Interleukin-11 (IL-11, thrombopoietin) is a pleiotropic 178-amino acid polypeptide with a molecular weight of $18 \mathrm{kDa}$ which is expressed by a wide range of mesenchymal tissues $(1,2)$ used in clinical practice as a megakaryocytopoiesis stimulator in patients with thrombocytopenia (3). It is well known that IL-11 protects the oral gastrointestinal mucosa against radiation (4) and 5-fluorouracil (5) damage by attenuating
TNF- $\alpha$ and IL- $1 \beta$ expression in hamsters. In attempt to elucidate this anti-inflammatory effect, we investigated if IL-11 could protect against ifosfamide (IFS)induced hemorrhagic cystitis (HC).

IFS is an alkylating agent from the oxazaphosphorine group with a broad spectrum of antineoplasic activity. In the absence of adequate uroprotection, patients treated with IFS for malignant tumors or immunosuppression develop a dose-limiting $\mathrm{HC}$ with an average incidence of $40 \%$. Such toxicity 
is attributed to the intravesical release of acrolein (ACR), an IFS highly urotoxic metabolite. It has been proposed that urothelial damage occurs by direct contact with ACR, causing edema, ulceration, neovascularization, hemorrhage and necrosis (6). Despite the preventive use of mesna, the occurrence of hematuria due to $\mathrm{HC}$ has been observed in $33 \%$ of patients (7). These facts increase the importance of studies to investigate novel therapies and elucidate the mechanisms involved in bladder lesion resulting from alkylating agent therapy.

Recombinant human IL-11 (rhIL-11, oprevelkin) has a number of biological activities which could impact $\mathrm{HC}$ including inhibition of pro-inflammatory cytokines such as tumor necrosis factor (TNF)$\alpha$ and interleukin-1 $\beta$ (IL-1 $\beta$ ), nitric oxide (NO) synthesis and apoptosis $(8,9)$, and stimulation of cell proliferation and differentiation and protection of connective tissue (8). For that reason, the present study investigated if rhIL-11 has a protective effect against IFS- induced $\mathrm{HC}$ in mice.

\section{MATERIALS AND METHODS}

Animals - Male Swiss mice (20-30 g), provided from the Central Bioterium of Pici, were kept in a temperature-controlled room with food ad libitum and water restriction 12 hours before they were sacrificed. All animal treatments and surgical procedures were performed in accordance with the Guide for Care and Use of Laboratory Animals, National Institutes of Health (Bethesda, MD, USA).

Drugs - Ifosfamide (IFS, Holoxane ${ }^{\circledR}$ : ASTA - AG, Frankfurt Germany; 1g), recombinant human interleukin-11 (rhIL-11, Neumega ${ }^{\circledR}$, Oprevelkin: Wyeth, $5 \mathrm{mg}$ ) and Evans blue (Sigma Chemical Co.) were dissolved in sterile saline. All other reagents were obtained from Sigma Chemicals Co (St. Louis, MO).

Effect of IL-11 on ifosfamide-induced hemorrhagic cystitis - Groups of 6 mice were pretreated with subcutaneous (s.c.) administration of sterile saline or rhIL-11 at 25,125 or $625 \mu \mathrm{g} 30$ minutes before the induction of $\mathrm{HC}$ with intraperitoneal injection of IFS at $400 \mathrm{mg} / \mathrm{kg}$. The control group received only sterile saline. Twelve hours after IFS treatment, ani- mals were euthanized, and the bladders were removed by careful dissection and emptied of urine. Bladder wet weight (BWW) was measured as a parameter of vesical edema and expressed as $\mathrm{g} / 20 \mathrm{~g}$ body weight (mean \pm SEM).

Macroscopic evaluation - Bladders were grossly examined for edema and hemorrhage. According to Gray's scoring criteria (12), edema was considered severe (3+) when fluid was seen externally and internally in the bladder walls, moderate $(2+)$ when confined to the internal mucosa, mild (1+) between normal and moderate and normal ( 0 ) when no edema was observed. Hemorrhage was scored as follows: $3+$, intravesical clots; $2+$, mucosal hematomas; $1+$, telangiectasia or dilatation of bladder vessels; and 0 , normal.

Microscopic evaluation - Bladders were fixed in formalin at $10 \%$, embedded in paraffin and processed for hematoxylin and eosin (HE, Reagen) staining. Histopathological analysis was performed by a person (GACB) who was unaware of the treatments and group divisions, and scored as follows: (0) normal epithelium and absence of inflammatory cell infiltration and ulceration, (1) mild changes involving reduction of urothelial cells, flattening with submucosal edema, mild hemorrhage and few ulcerations, and (2) severe changes including mucosal erosion, inflammatory cell infiltration, fibrin deposition, hemorrhage and multiple ulcerations (10).

Evans blue extravasation assay - Vesical vascular permeability was evaluated by the Evans blue extravasation technique. Following the same previous group division $(n=6)$ and protocol, $2.5 \%$ Evans blue $(25 \mathrm{mg} / \mathrm{kg})$ was injected intravenously via the retro orbital plexus 30 minutes before the animals were sacrificed. Bladders were then excised, dissected and placed into glass tubes containing a formamide solution $(1 \mathrm{~mL} / \mathrm{bladder})$ at $56^{\circ} \mathrm{C}$ overnight to extract the stain. The total extracted dye was determined by measuring the absorbance change at $630 \mathrm{~nm}$ (ELISA). At the same time, an absorbance-concentration curve was determined. The results were then reported as in micrograms of Evans blue per bladder (mean \pm SEM).

Statistical analysis - Data were reported as the mean \pm SEM (bladder wet weight, Evans blue extravasation and NOS activity) or the median values 
followed by range values (macroscopic and histopathological scores) of groups of six mice. Statistical analysis was performed using one way analysis of variance (ANOVA) followed by Newman-Keuls test, when appropriate. Macroscopic and microscopic scores were evaluated by Kruskal Wallis non-parametric test followed by Dunn's multiple comparison. Statistical significance was set at $\mathrm{p}<0.05$.

\section{RESULTS}

Protective effect of IL-11 against ifosfamideinduced increase in bladder wet weight - IFS at 400 $\mathrm{mg} / \mathrm{kg}$ i.p. induced a marked increase $(51.40 \pm 7.10$ $\mathrm{g} / 20 \mathrm{~g}, 211.51 \%$ increase, $\mathrm{p}<0.05)$ in bladder wet weight 12 hours after its administration when compared to the control group which received only sterile saline $(16.50 \pm 0.40 \mathrm{~g} / 20 \mathrm{~g})$. In a dose-dependent man- ner, rhIL-11 provided partial prevention (Figure-1) of the bladder wet weight increase induced by IFS, reaching a maximal effect at $125 \mu \mathrm{g}(38.32 \pm 2.89 \mathrm{~g} /$ $20 \mathrm{~g}, 37.48 \%$ reduction, $\mathrm{p}<0.05)$ and $625 \mu \mathrm{g}(35.54 \pm$ $2.67 \mathrm{~g} / 20 \mathrm{~g}, 45.44 \%$ reduction, $\mathrm{p}<0.05$ ). The effect of rhIL-11 at $25 \mu \mathrm{g}$ did not reach statistical significance $(42.28 \pm 0.93 \mathrm{~g} / 20 \mathrm{~g}, 26.13 \%$ reduction, $\mathrm{p}$ > $0.05)$.

Protective effect of IL-11 against ifosfamideinduced macroscopic and microscopic changes - As demonstrated in Table-1, bladders from animals treated with IFS only developed significant edema $($ median $=$ $2, \mathrm{p}<0.05)$ and hemorrhage (median $=2, \mathrm{p}<0.05)$, when compared to the control group (median $=0$ for both parameters). rhIL-11 at $625 \mu \mathrm{g}$ significantly protected ifosfamide- induced gross edema and hemorrhage (median $=1, \mathrm{p}<0.05$, for both parameters). Table-1 also shows that IFS induced microscopic alterations, when compared to the control bladders, such

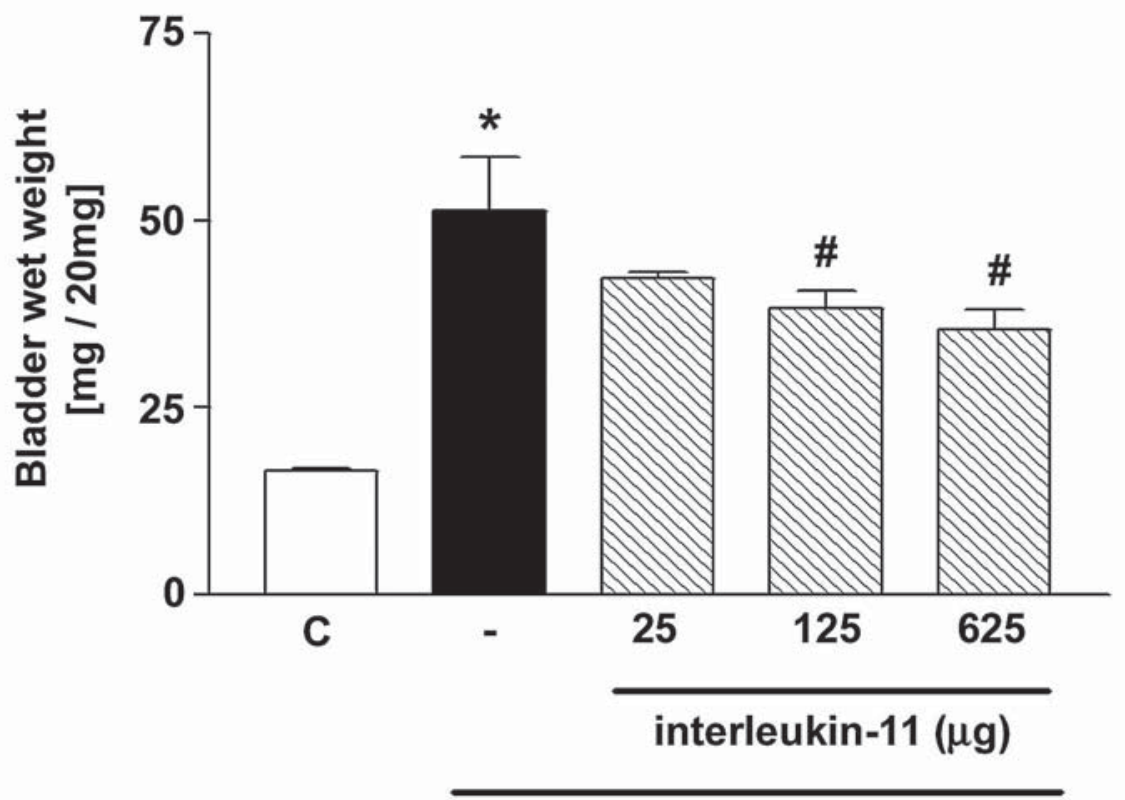

ifosfamide $400 \mathrm{mg} / \mathrm{kg}$

Figure 1 - Protective effect of rhIL-11 against ifosfamide-induced bladder wet weight increase. Ifosfamide at $400 \mathrm{mg} / \mathrm{kg}$ induced an increase in BWW when compared to the control group $(C)$, and rhIL-11 at $125 \mu \mathrm{g}$ and $625 \mu \mathrm{g}$ protected against this side effect. * $p<$ 0.05 compared with control group. \# $p<0.05$ compared to ifosfamide only group. ANOVA followed by Newman-Keuls. 
Table 1 - Macroscopic and microscopic analysis of rhIL-11 effect on ifosfamide-induced hemorrhagic cystitis.

\begin{tabular}{lccc}
\hline Experimental Groups & $\begin{array}{c}\text { Macroscopic Analysis } \\
\text { (edema) }\end{array}$ & $\begin{array}{c}\text { Macroscopic Analysis } \\
\text { (hemorrhage) }\end{array}$ & Microscopic Analysis \\
\hline C & $0(0-0)$ & $0(0-0)$ & $0(0-0)$ \\
IFS & $2(1-3)^{\mathrm{a}}$ & $2(1-3)^{\mathrm{a}}$ & $2(2-2)^{\mathrm{a}}$ \\
IL-11 25 & $2(1-3)$ & $2(1-3)$ & $1(1-2)$ \\
IL-11 125 & $2(1-2)$ & $2-3(1-3)$ & $2(1-2)$ \\
IL-11 625 & $1(0-1)^{\mathrm{b}}$ & $1(0-2)^{\mathrm{b}}$ & $1(1-1)^{\mathrm{b}}$ \\
\hline
\end{tabular}

Ifosfamide (400 mg/kg, i.p.) induced macroscopic (edema and hemorrhage) and microscopic alterations (fibrin deposition, edema, urothelial desquamation, hemorrhage and leukocyte infiltration). Pretreatment with rhIL-11 at $625 \mu \mathrm{g}$ protected against those side effects at $625 \mu \mathrm{g}$. C, control group treated with saline alone; IFS, ifosfamide only treated group; rhIL-11 25, 125 and 625, pretreated groups that received 25,125 and $625 \mu \mathrm{g}$ of rhIL-11, respectively. The results are reported as medians and range $(n=6) .{ }^{a} p<0.05$ compared to the control group. ${ }^{b} p<0.05$ compared to the IFS group. Kruskal-Wallis and Dunn's test.

as edema, hemorrhage, fibrin deposition, neutrophil infiltration and vascular congestion (median $=2, \mathrm{p}<$ 0.05). rhIL-11 at $625 \mu \mathrm{g}$ partially prevented these
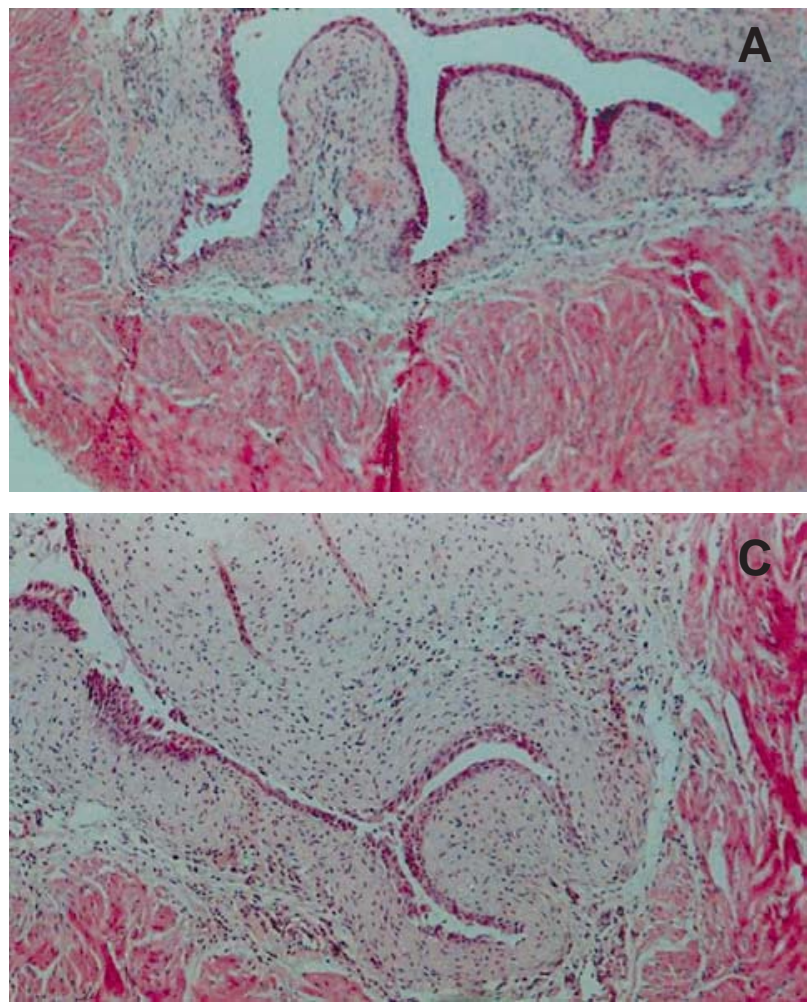

side effects (median $=1, \mathrm{p}<0.05)$ (Table-1, Figure2). In macroscopic and microscopic evaluation, rhIL11 at 25 and $125 \mu \mathrm{g}$ did not reach statistical differ-

Figure 2 - Histological analysis of cross sections of representative bladder walls. A) Control group which received only saline. B) Ifosfamide at $400 \mathrm{mg} / \mathrm{kg}$ induced urothelium desquamation, edema, pronounced hemorrhage and fibrin deposition and leukocyte infiltration when compared to control, shown in A. C) and D) Pretreatment with rhIL-11 at $625 \mu \mathrm{g}$ was capable of reducing these side effects. (HE, $A, B$ and $C X 100$ and $D X 400$ ). 
ence compared of the group that received only IFS $(\mathrm{p}>0.05)$.

Protective effect of IL-11 against ifosfamideinduced increase of Evans blue extravasation in bladder - IFS at $400 \mathrm{mg} / \mathrm{kg}$ induced significant Evans blue extravasation in the bladders $(6.08 \pm 1.27 \mu \mathrm{g} /$ bladder, $4576.92 \%$ increase, $\mathrm{p}<0.05)$ when compared to control group $(0.13 \pm 0.12 \mu \mathrm{g} /$ bladder $)$. rhIL- 11 in a dose-dependent fashion prevented the IFS- induced Evans blue extravasation (Figure-3), maximally at 125 $\mu \mathrm{g}(2.37 \pm 0.59 \mu \mathrm{g} /$ bladder, $62.35 \%$ reduction, $\mathrm{p}<$ $0.05)$ and $625 \mu \mathrm{g}(2.72 \pm 0.41 \mu \mathrm{g} / \mathrm{bladder}, 56.47 \%$ reduction, $\mathrm{p}<0.05)$. rhIL-11 at $25 \mu \mathrm{g}$ did not reach statistical significance $(3.79 \pm 0.52 \mu \mathrm{g} /$ bladder, $\mathrm{p}>$ $0.05)$.

\section{COMMENTS}

The present study demonstrates the protective effect of rhIL-11 on IFS-induced hemorrhagic cystitis (HC). Our results show evidence that pretreatment with rhIL-11 in a dose-dependent manner prevents the increase of bladder wet weight, (parameter used to measure edema) and Evans blue extravasation in bladder (parameter used to measure vascular permeability), induced by IFS at $400 \mathrm{mg} / \mathrm{kg}$. The results obtained were confirmed by macroscopic and microscopical analysis, in which only $625 \mu \mathrm{g}$, but not $125 \mu \mathrm{g}$ dose, was effective in protecting against IFSinduced macroscopic edema and hemorrhage and microscopic alterations. Consequently, our preclinical

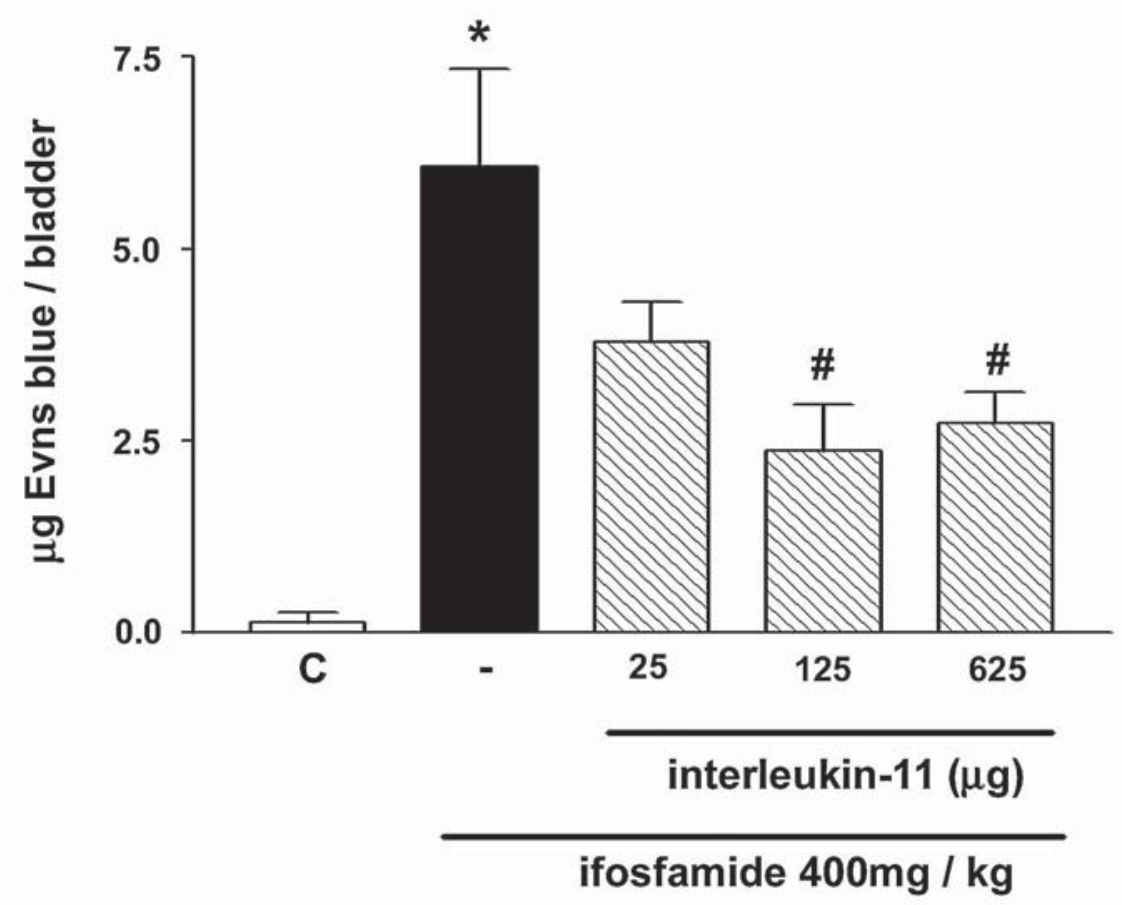

Figure 3 - Protective effect of rhIL-11 against ifosfamide-induced Evans blue extravasation in bladder. Ifosfamide at $400 \mathrm{mg} / \mathrm{kg}$ induced an increase in Evans blue extravasation when compared to the control group $(C)$, thus demonstrating an increase in vascular permeability. rhIL-11 at $125 \mu \mathrm{g}$ and $625 \mu \mathrm{g}$ reduced ifosfamide-induced Evans blue extravasation. $* p<0.05$ compared with control group. \# $p<$ 0.05 compared to ifosfamide only group. ANOVA followed by Newman-Keuls. 
study suggests a previously unreported effect of rhIL11 for the treatment of experimental ifosfamide-induced hemorrhagic cystitis.

Recently, some important mechanisms of experimental ifosfamide-induced hemorrhagic cystitis have been elucidated. Pro-inflammatory cytokines such as platelet-activating factor (PAF), TNF- $\alpha$ and IL- $1 \beta$ have been implicated in its pathogenesis. Previously, we demonstrated the participation of interleukin-1 $\beta$ (IL-1 $\beta$ ) and tumor necrosis factor- $\alpha$ $(\mathrm{TNF}-\alpha)$ pathway in the induction of nitric oxide (NO) production as an important pathway in the pathogenesis of lesion in hemorrhagic cystitis induced by alkylating agents. NO was demonstrated to be the final mediator of urothelial damage and hemorrhage in that type of HC (11). TNF- $\alpha$ and IL$1 \beta$ have been shown to be important mediators of NO synthesis, since treatment with anti-TNF- $\alpha$ and anti-IL-1 $\beta$ decreased cyclophosphamide vesical damage as well the rise of inducible NO synthase expression and activity $(12,13)$.

A previous study from our laboratory proved that dexamethasone alone was ineffective in preventing experimental IFS-induced hemorrhagic cystitis, but when combined with mesna, this corticosteroid potentiated its protective effect (14). More recently, we showed that amifostine and glutathione prevent ifosfamide and acrolein-induced hemorrhagic cystitis in mice (15). Therefore, it is becoming important to establish novel therapies for hemorrhagic cystitis, for the reason that, as previously demonstrated, $33 \%$ of patients using alkylating agents for the treatment of cancer develop hematuria (hemorrhagic cystitis sign) despite the preventive use of mesna (7). An experimental study in rats reinforced that the notion that there a high percentage of non protection despite preventive mesna usage (14).

Interleukin-11 (IL-11) has been extensively investigated as a protective agent for inflammatory events, such as inflammatory bowel disease, psoriasis, autoimmune joint disease, and many other inflammatory diseases (16). rhIL-11 has been shown to down regulate macrophage production in vitro of IL-1 $\beta$, TNF- $\alpha$, IL-12 and nitric oxide, without inducing antiinflammatory cytokines such as IL-10, TGF-b, and IL-6 (8). It was also observed that rhIL-11 attenuates
Th1 cytokines production in human psoriasis lesions, noting that its effect is not restricted to macrophages (17). More recent works demonstrate the rhIL-11 property of inhibiting NF- $\kappa \mathrm{B}$ and AP-1 activation in islets to prevent streptozotocin-induced diabetes (18). Other studies have successfully used IL-11 for the prevention of intestinal ischemia-reperfusion lesion (19) and intestinal mucosa damage in response to chemotherapy and radiation due to diminished cell apoptosis and consequent death (9). On the other hand, other authors demonstrated that IL-11 does not prevent methotrexate-induced intestinal cell apoptosis but reduces the damage by compensatory crypt cell proliferation (20). Our data introduce a novel anti-inflammatory effect of IL-11 and are in accordance with previous reports in the literature.

In conclusion, the present study demonstrated that the pleiotropic cytokine rhIL-11 partially prevents IFS-induced experimental $\mathrm{HC}$, an inflammatory event that depends on TNF- $\alpha$, IL-1 $\beta$ and nitric oxide release. Possibly, rhIL-11 acts on an inhibitory pathway of these inflammatory mediators. Taking into account that rhIL-11 is already being used in clinical practice, it is possible to propose a clinical trial to investigate its effect on human HC.

\section{ACKNOWLEDGMENTS}

Supported by grant from CNPq, Ministry of Technology, Brazil. Maria Silvandira Freire and José Ivan Rodrigues, from Federal University of Ceará, provided technical support. Dr. A. Leyva edited the manuscript.

\section{CONFLICT OF INTEREST}

None declared.

\section{REFERENCES}

1. Neben S, Turner K: The biology of interleukin 11. Stem Cells. 1993; 11: 156-62.

2. Leng SX, Elias JA: Interleukin-11. Int J Biochem Cell Biol. 1997; 29: 1059-62. 
3. Basser R: The impact of thrombopoietin on clinical practice. Curr Pharm Des. 2002; 8: 369-77.

4. Sonis ST, Peterson RL, Edwards LJ, Lucey CA, Wang L, Mason L, et al.: Defining mechanisms of action of interleukin-11 on the progression of radiation-induced oral mucositis in hamsters. Oral Oncol. 2000; 36: 37381.

5. Sonis ST, Van Vugt AG, McDonald J, Dotoli E, Schwertschlag U, Szklut P, et al.: Mitigating effects of interleukin 11 on consecutive courses of 5-fluorouracil-induced ulcerative mucositis in hamsters. Cytokine. 1997; 9: 605-12.

6. Cox PJ: Cyclophosphamide cystitis-identification of acrolein as the causative agent. Biochem Pharmacol. 1979; 28: 2045-9.

7. Shepherd JD, Pringle LE, Barnett MJ, Klingemann HG, Reece DE, Phillips GL: Mesna versus hyperhydration for the prevention of cyclophosphamide-induced hemorrhagic cystitis in bone marrow transplantation. J Clin Oncol. 1991; 9: 2016-20.

8. Trepicchio WL, Bozza M, Pedneault G, Dorner AJ: Recombinant human IL-11 attenuates the inflammatory response through down-regulation of proinflammatory cytokine release and nitric oxide production. J Immunol. 1996; 157: 3627-34.

9. Orazi A, Du X, Yang Z, Kashai M, Williams DA: Interleukin-11 prevents apoptosis and accelerates recovery of small intestinal mucosa in mice treated with combined chemotherapy and radiation. Lab Invest. 1996; 75: 33-42.

10. Gray KJ, Engelmann UH, Johnson EH, Fishman IJ: Evaluation of misoprostol cytoprotection of the bladder with cyclophosphamide (Cytoxan) therapy. J Urol. 1986; 136: 497-500.

11. Souza-Fiho MV, Lima MV, Pompeu MM, Ballejo G, Cunha FQ, Ribeiro Rde A: Involvement of nitric oxide in the pathogenesis of cyclophosphamide-induced hemorrhagic cystitis. Am J Pathol. 1997; 150: 247-56.

12. Ribeiro RA, Freitas HC, Campos MC, Santos CC, Figueiredo FC, Brito GA, et al.: Tumor necrosis factoralpha and interleukin-1beta mediate the production of nitric oxide involved in the pathogenesis of ifosfamide induced hemorrhagic cystitis in mice. J Urol. 2002; 167: 2229-34.

13. Gomes TN, Santos CC, Souza-Filho MV, Cunha FQ, Ribeiro RA: Participation of TNF-alpha and IL-1 in the pathogenesis of cyclophosphamide-induced hemorrhagic cystitis. Braz J Med Biol Res. 1995; 28: 1103-8.

14. Vieira MM, Brito GA, Belarmino-Filho JN, Macedo FY, Nery EA, Cunha FQ, et al.: Use of dexamethasone with mesna for the prevention of ifosfamide-induced hemorrhagic cystitis. Int J Urol. 2003; 10: 595-602.

15. Batista CK, Mota JM, Souza ML, Leitao BT, Souza MH, Brito GA, et al.: Amifostine and glutathione prevent ifosfamide- and acrolein-induced hemorrhagic cystitis. Cancer Chemother Pharmacol. 2006; Epub ahead of print.

16. Schwertschlag US, Trepicchio WL, Dykstra KH, Keith $\mathrm{JC}$, Turner KJ, Dorner AJ: Hematopoietic, immunomodulatory and epithelial effects of interleukin11. Leukemia. 1999; 13: 1307-15.

17. Trepicchio WL, Ozawa M, Walters IB, Kikuchi T, Gilleaudeau P, Bliss JL, et al.: Interleukin-11 therapy selectively downregulates type I cytokine proinflammatory pathways in psoriasis lesions. J Clin Invest. 1999; 104: 1527-37. Erratum in: J Clin Invest 2000; 105: 396.

18. Lgssiar A, Hassan M, Schott-Ohly P, Friesen N, Nicoletti F, Trepicchio WL, et al.: Interleukin-11 inhibits NF-kappaB and AP-1 activation in islets and prevents diabetes induced with streptozotocin in mice. Exp Biol Med (Maywood). 2004; 229: 425-36.

19. Kuenzler KA, Pearson PY, Schwartz MZ: IL-11 pretreatment reduces cell death after intestinal ischemiareperfusion. J Surg Res. 2002; 108: 268-72.

20. Gibson RJ, Keefe DM, Thompson FM, Clarke JM, Goland GJ, Cummins AG: Effect of interleukin-11 on ameliorating intestinal damage after methotrexate treatment of breast cancer in rats. Dig Dis Sci. 2002; 47: 2751-7.

Accepted after revision: December 15, 2006 